\title{
Epidemiologie und Diagnostik der Gonarthrose
}

\author{
$\square$ Jörg Lützner, Stephan Kirschner, Klaus-Peter Günther
}

\section{Zusammenfassung}

Die Gonarthrose ist eine multifaktoriell bedingte Erkrankung die etwa $10 \%$ der europäischen Bevölkerung betrifft. Bei nicht identifizierbaren Risikofaktoren handelt es sich um eine „idiopathische“ Gonarthrose. Bestehen konstitutionelle, mechanische oder metabolisch-endokrine Riskofaktoren spricht man von einer „sekundären“ Gonarthrose. Die Diagnostik besteht neben Anamnese und klinischer Untersuchung in erster Linie in Röntgenaufnahmen in 2 Ebenen, wobei ie a.p.Aufnahme unter Belastung im Stand erfolgen sollte. Die röntgenologische Einteilung des Schweregrades erfolgt am häufigsten nach dem Kellgren \& Lawrence-Score.

\section{Epidemiology and Diagnostics of Osteoarthrosis}

Osteoarthritis of the knee is a disease with a multifactorial aetiology. About $10 \%$ of the Caucasian population are affected by osteoarthritis of the knee. In those cases where there are no identifiable risk factors it is named primary "idiopathic" osteoarthritis. In cases where there are known mechanical or metabolic risk factors it is called "secondary" osteoarthritis. Diagnostic work-up should include a clinical examination and X-rays (weight-bearing anteroposterior view). The Kellgren and Lawrence score is highly useful for grading the severity of the disease.

\section{Definition und Klassifikation von Arthrosen}

Mit den Begriffen „Arthrose“ oder „Osteoarthrose“ wird ein Erkrankungsprozess in Gelenken bezeichnet, der unterschiedliche Ursachen haben kann, aber letztlich in einen gemeinsamen biologischen, morphologischen und klinischen Verlauf mündet. Die im Mittelpunkt stehende Knorpeldegeneration führt von einer beginnenden Fibrillation bis zur vollständigen Zerstörung der Gelenkoberfläche. Es ist jedoch nicht nur der hyaline Knorpel von den Veränderungen betroffen, sondern mit ihm alle am Gelenkaufbau beteiligten Strukturen (subchondraler Knochen, Bänder, Kapsel, Synovialmembran und Muskeln).

Sämtliche Gelenke im Körper können von einer Arthrose befallen sein. In Abhängigkeit von Anzahl und Kombina-

OP-JOURNAL 2006; 22: 142-147

(c) Georg Thieme Verlag KG Stuttgart · New York tion betroffener Gelenkregionen sowie der Schädigungsursache werden unterschiedliche Erkrankungsformen abgegrenzt (Tab.1): Lassen sich keine Risikofaktoren für die Entstehung der $\mathrm{Er}$ krankung definieren, spricht man von idiopathischen Arthrosen. Bestehen jedoch Gelenkverletzungen in der Anamnese, erkennbare Formstörungen (z.B. ausgeprägte Achsdeformitäten) oder systemische Erkrankungen als mögliche Ursache der Knorpelschädigung, handelt es sich um eine sekundäre Arthrose.

In der Gruppe der idiopathischen Arthrosen werden zwei Erkrankungsformen unterschieden: Lassen sich die Veränderungen nur an ein oder maximal zwei Gelenken nachweisen, spricht man von lokalisierter Arthrose. Sind drei oder mehrere Gelenkregionen betroffen (unter Einschluss der Hände), handelt es sich um eine generalisierte Arthrose. Dieses Konzept berücksichtigt die Tatsache, dass Menschen - möglicherweise auch genetisch beeinflusst - unterschiedliche Toleranzgrenzen für die Entwicklung und
Ausbreitung degenerativer Gelenkerkrankungen aufweisen [1]. Generalisierte Arthrosen sind jedoch nicht nur bei der Gonarthrose zu beobachten, wie früher häufig angenommen wurde, sondern auch Patienten mit Koxarthrose oder Befall anderer Gelenke können davon betroffen sein [10].

\section{Ätiologie der Gonarthrose}

Der genannten Einteilung in „idiopathische“ und „sekundäre“ Erkrankungsformen liegt die Hypothese zugrunde, dass sich eindeutige Ursachen für eine Arthrose definieren lassen. Neuere ätiologische Konzepte gehen jedoch davon aus, dass es sich um einen sehr komplexen Erkrankungsprozess handelt, bei dem zwar ganz unterschiedliche Schäden auf das Gelenk einwirken können, aber letztlich die Entstehung und der Verlauf einer Arthrose von vielen Faktoren abhängen, die heute nur zum Teil bekannt sind. Dies gilt auch für das Kniegelenk: Auf der einen Seite lassen sich Risikofaktoren definieren, die zu einer Knorpelschädigung führen können, und auf der anderen Seite wirken kontinuierlich ablaufende Reparaturmechanismen auf Gelenkebene der Zerstörung entgegen. Letztlich entscheidet das Überwie-

Tab. 1 Klassifikation der Arthrose (modifiziert nach Brandt et al. 1986) [5]

\begin{tabular}{l} 
Idiopathische Arthrose \\
\hline Lokalisiert \\
\hline Generalisiert \\
\hline Sekundäre Arthrose \\
\hline Posttraumatisch \\
\hline $\begin{array}{l}\text { Angeborene und erworbene Gelenk- } \\
\text { schäden }\end{array}$ \\
\hline Metabolische Störungen \\
\hline Endokrine Erkrankungen \\
\hline Sonstige Ursachen
\end{tabular}


gen der schädigenden oder reparativen Einflüsse darüber, ob die Erkrankung „kompensiert“ bleibt oder sich eine symptomatische Arthrose ausbildet (Abb.1).

Unterschiedliche Risikofaktoren können zu einer Arthroseentstehung am Kniegelenk beitragen [11]:

- Konstitutionelle Risikofaktoren bedingen gewissermassen die „Empfänglichkeit" des Patienten für die Entwicklung eines Knorpelschadens. Dies betrifft vor allem Alter und Geschlecht, denn mit steigendem Lebensalter nimmt die Häufigkeit von Arthrosen $\mathrm{zu}$, und Frauen leiden nach der Menopause mehr an Gonarthrosen als Männer. Wenn die Gonarthrose mit degenerativen Veränderungen der Hand- und Fingergelenke gemeinsam beobachtet wird, spricht man von generalisierter Arthrose mit vermutlich genetischer Disposition.

- MechanischeRisikofaktoren:Hackenbroch [12] beschrieb 1943 in seinem Konzept der „präarthrotischen Deformität" den Weg vom Gelenkschaden, der als unmittelbare Folge einer Vorerkrankung zurückbleibt und dann aufgrund der persistierenden Formstörung - mehr oder weniger rasch zur Arthrose führt. Aufgrund des heute multifaktoriell betrachteten Prozesscharakters ist dieses Konzept zwar in den Hintergrund gerückt, doch lassen sich eindeutige mechanische
Risikofaktoren definieren: Dazu gehören akute Gelenkverletzungen (Frakturen mit Kniegelenkbeteiligung und daraus resultierender direkter Knorpelschädigung oder sekundärer Schädigung aufgrund einer posttraumatischen Deformität, Bandverletzungen mit der Folge einer Instabilität), eine chronische Gelenkschädigung infolge beruflicher oder sportlicher Überlastung und angeborene oder erworbene Gelenkerkrankungen (Osteochondrosis dissecans und skeletale Dysplasien wie z.B. die epiphysäre Dysplasie). Auch können Achsabweichungen der unteren Extremitäten (z. B. ausgeprägtes Genu varum oder valgum) und Hypermobilitäts-Syndrome langfristig zur Entstehung einer Gonarthrose führen, auch wenn gerade hier die Grenze zwischen physiologischem Befund und Krankheitswert oft fließend ist. Gemeinsam ist all diesen Risikofaktoren, dass es im Lauf der Zeit aufgrund gestörter Krafteinleitung zu einem Missverhältnis zwischen erhöhter mechanischer Belastung des Gelenkknorpels und seiner physiologischen Belastbarkeit mit einer daraus resultierenden Knorpeldegradation kommt.

- Metabolische/endokrine Störungen: Bei unterschiedlichen metabolischen Erkrankungen kann die Ablagerung von Stoffwechselprodukten im Gelenkknorpel die Belastbarkeit reduzieren (z.B. Ochronose, Hämochromatose). Für weitere Risikofaktoren konnte

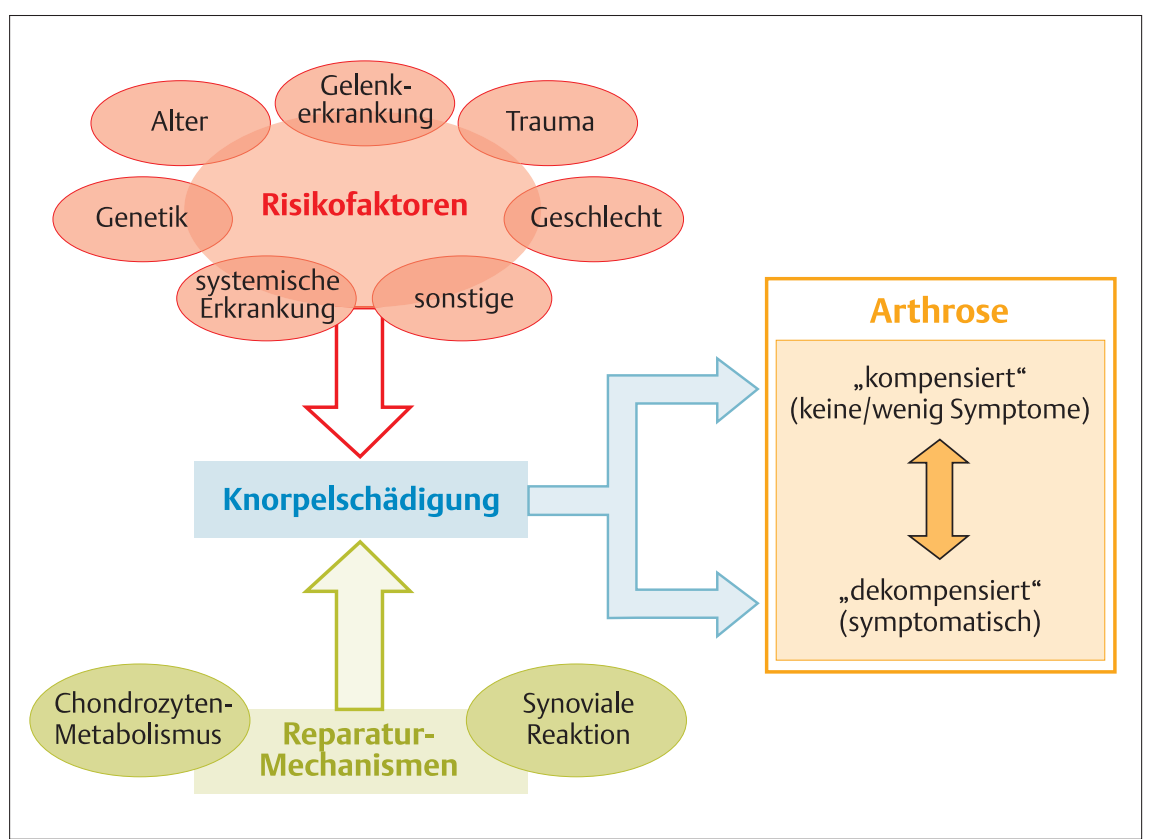

Abb. 1 Die Arthrose als Erkrankungsprozess (modifiziert nach Brandt et al. 1998) [4]. ebenfalls ein Zusammenhang mit Arthrosen der Kniegelenke und Hüftgelenke nachgewiesen werden. Dazu gehören z.B. Diabetes mellitus und Hypercholesterinämie [16, 17]. Auch für Übergewicht bzw. Adipositas ist eine Assoziation mit der Entstehung von Gonarthrosen belegt $[8,18]$. Dieser Risikofaktor nimmt jedoch insofern eine Sonderstellung ein, dass er sowohl über mechanische Einflüsse (dauerhafte statische Überlastung) als auch systemische Faktoren (stoffwechselbedingte Ernährungsstörung von Chondrozyten) wirksam sein kann.

- Sonstige Risikofaktoren: Entzündliche Gelenkerkrankungen (rheumatische oder septische Arthritis) und die Ablagerung von Kalziumpyrophosphat- bzw. Apatit-Kristallen können ebenfalls zu einer sekundären Knorpelschädigung führen. Bei den entzündlichen Gelenkerkrankungen ist dies auf eine direkte Schädigung des Knorpels durch degradierende Enzyme und synoviale Pannusbildung zurückzuführen. Bei Kristallablagerungen ist dagegen noch unsicher, inwieweit z. B. die Chondrokalzinose (Ablagerung von Kalziumpyrophosphat in Gelenkknorpel und Meniskus) als eine mögliche Ursache oder aber Folgeerscheinung der Arthrose zu werten ist. Neurologische Erkrankungen können aufgrund einer Störung der Tiefensensibilität mit schwersten Gonarthrosen einhergehen (neuropathische $\mathrm{Ar}$ thropathie).

Lassen sich Risikofaktoren für die Knorpelschädigung am Kniegelenk identifizieren, spricht man von sekundären Arthrosen.

Die Arthrose ist jedoch eine multifaktoriell beeinflusste Erkrankung: Bekannte und unbekannte Ursachen führen zwar zum initialen Knorpelschaden, aber der weitere Verlauf hängt von genetischen, konstitutionellen, metabolischen, endokrinen und mechanischen Faktoren ab.

\section{Pathomorphologie}

Eine Arthrose entsteht dann, wenn das normale Gleichgewichts von anabolen und katabolen Prozesen im Gelenkknorpel gestört ist. Gelenkknorpel besteht aus Chondrozyten, der von ihnen synthetisierten extrazellulären Matrix und Wasser. Matrixproteine (Proteoglykane) binden das Wasser und werden durch Kollagenfasern in einem komplexen Netzwerk gehalten, das für die Visko- 
elastizität des Knorpels verantwortlich ist. Um unter mechanischer Belastung diese charakteristischen Dämpfungseigenschaften auch längerfristig sicherzustellen, muss sich Gelenkknorpel kontinuierlich erneuern. In einem sehr langsam ablaufenden Prozess werden Matrixbestandteile - gesteuert von Zytokinen, Wachstumsfaktoren und Matrixmetalloproteinasen (MMP) - gleichzeitig abgebaut und neu synthetisiert.

Bei der Arthrose gerät diese Balance von anabolen und katabolen Prozessen aus dem Gleichgewicht und der Knorpelabbau (Degradation) überwiegt. Eine v.a. durch Interleukin-1 (IL-1) und TNF- $\alpha$ stimulierte Synthese der Matrixmetalloproteinasen führt zum Abbau von Interzellularsubstanz. Gleichzeitig ablaufende reparative Prozesse können diese Knorpelzerstörung jedoch nicht mehr kompensieren [6] und es kommt zu makroskopisch erkennbaren Veränderungen, die auch im Röntgenbild fassbar werden:

- Gelenkspaltverschmälerung: Abbau von Knorpelmasse.

- Subchondrale Spongiosaverdichtung: Erhöhte Kalksalzeinlagerung im Knorpelnahen Knochen aufgrund veränderter Knorpeldämpfungseigenschaften.

- Osteophyten: Am Rand der Gelenkfläche kann die fehlgesteuerte Reparationsleistung von Chondrozyten zur Bildung von Osteophyten führen.

- Geröllzysten: Mikrofrakturen im subchondralen Knochen heilen nicht adäquat aus, da ein Einstrom von Synovialflüssigkeit Reparationsvorgänge behindert und zur bindegewebigen Abkapselung führt.

\section{Häufigkeit der Gonarthrose}

Insgesamt gehört die Arthrose zu den häufigsten Erkrankungen in Industrienationen. Der Befall von Hand, Wirbelsäule, Hüft- und Kniegelenk sowie Großzehengrundgelenk steht gegenüber degenerativen Veränderungen an anderen Gelenken weit im Vordergrund. Gemäß unserer funktionellen Beanspruchung besteht vor allem für die lasttragenden Gelenke der unteren Extremität (Knie und Hüfte) sowie die in Greiffunktion involvierten Gelenke der Hand (PIP- und DIP-Gelenke, CMC-Gelenk des ersten Strahls) eine Arthrose-Prädilektion.

Viele Menschen weisen die röntgenologischen Zeichen degenerativer Gelenker- krankungen auf, ohne über entsprechende Beschwerden zu klagen. Deshalb muss bei Angaben zur Häufigkeit der Gonarthrose differenziert werden, ob der Befall nur im Röntgenbild sichtbar ist oder auch eine klinisch relevante Erkrankung besteht. [19]

In einer niederländischen Studie [20] wurde festgestellt, dass 15\% der Bevölkerung im 50. Lebensjahr und 50\% der Personen im 80. Lebensjahr die röntgenologischen Zeichen einer Gonarthrose aufweisen. Da nur etwa ein Drittel dieser Personen auch entsprechende Beschwerden angibt, kann man davon ausgehen, dass in Europa etwa 10\% der gesamten Bevölkerung unter einer Gonarthrose leiden. Diese Häufigkeit ist - gerade auch vor dem Hintergrund einer altersabhängigen Zunahme des Arthrosebefalls - von großer Bedeutung für gesundheitsökonomische Diskussionen. In Deutschland werden jährlich mehr als $300000 \mathrm{~Pa}$ tienten mit etwa 5 Mio. Krankenhaustagen aufgrund einer Arthrose stationär behandelt und die Hälfte dieser Fälle betrifft Patienten mit Gonarthrose [9]. Etwa 120000 Knie-Endoprothesen werden in Deutschland pro Jahr wegen einer fortgeschrittenen Gonarthrose implantiert [3].

\section{Diagnostik}

Betroffene Patienten klagen in der Regel über Schmerzen, Steifigkeit und Funktionsstörungen.

Am Anfang sind dies meist Belastungsschmerzen, im fortgeschrittenen Stadium kommen nächtliche Schmerzen und „Anlaufschmerzen“ gerade bei der Gonarthrose hinzu. Die Schmerzen können auf entzündliche Ursachen (Synovialitis, Gelenkerguss, Bursitis) oder mechanische Ursachen (Blockierung, PeriostDehnung, subchondrale Mikrofrakturen) zurückgeführt werden. Zusätzlich tragen auch eine intraossäre Druckerhöhung (Nachtschmerz) und die Verspannung der gelenknahen Muskulatur zum Beschwerdebild bei. Generell gilt jedoch, dass Schmerzen häufig nicht mit dem Schweregrad röntgenologisch nachweisbarer Veränderungen korrelieren.

Die meist morgendliche Steifigkeit dauert in der Regel weniger als 30 Minuten. Diese begrenzte Zeit ist ein Unterscheidungsmerkmal zu rheumatischen Erkrankungen, bei denen in der Regel eine deutlich länger anhaltende Morgensteifigkeit angegeben wird.
Funktionsstörungen betreffen vor allem die Fortbewegung (Einschränkung der Gehstrecke sowie der Benutzung von PKW und öffentlichen Nahverkehrsmitteln), Aktivitäten in der Körperpflege (z.B. Waschen, Ankleiden) und sportliche Tätigkeiten. Beim Vorliegen einer Arthrose führen Schmerzen und Funktionseinschränkung häufig zu Störungen der sozialen Interaktion und dem Verlust an gesundheitsbezogener Lebensqualität.

\section{Klinische Untersuchung}

- Anamnese: Detaillierte Abfrage von Beschwerden, Verletzungen, Vorerkrankungen und -operationen, Berufsund Sportbelastung sowie bisher durchgeführten Therapiemaßnahmen (u.a. Medikamente, Hilfsmittel). Arthrosespezifische Klassifikationssysteme wie z.B. der WOMAC-Score [2] erfassen zuverlässig das Ausmaß von Schmerzen, Steifigkeit und Funktionsstörungen.

- Inspektion: Beobachtung von Gangbild (Schmerz- und Schonhinken) und Statik (z.B. Beckenschiefstand bei Kniebeugekontraktur oder Achsdeformität). Dokumentation der Muskelumfänge (Hinweis auf Schonung des betroffenen Beins).

- Palpation: Gezielte Prüfung zugänglicher Weichteil- und Knochenstrukturen (Ausschluss von Insertionstendinopathie, Bursitis und Muskelverspannung). Prüfung auf Vorliegen von Gelenkschwellungen, denen u.a. ein Erguss (Prüfung des Phänomens der „tanzenden Patella“), eine Synovialitis oder die Ausbildung von Osteophyten zugrunde liegen kann. Auch Meniskusganglien oder eine Poplitealzyste können zu gelenknahen Schwellungen führen. Die retropatellaren Knorpelflächen sind zumindest teilweise einer Palpation zugänglich.

- Beweglichkeitsprüfung: Differenzierte Erhebung des aktiven und passiven Bewegungsumfanges nach der Neutral-0-Methode, Dokumentation von Krepitation und Schmerzen. Beachtung von häufig mit fortgeschrittener Gonarthrose einhergehenden Beugekontrakturen.

- Funktionstests: Prüfung von „Meniskuszeichen“ und Bandstabilität (Schädigungen der Kreuzbänder und Seitenbänder können sowohl in der Entstehung von Gonarthrosen eine Rolle spielen als auch bei fortgeschrittener Gelenkdestruktion sekundär auftreten). Das häufig in der Literatur beschriebene „Zohlen-Zeichen“ als mög- 
licher Hinweis auf retropatellare Knorpelschäden ist nach unserer Auffassung wenig sensitiv.

Da Schmerzen in Gelenkregionen neben einer Arthrose auch andere Ursachen haben können (z.B. fortgeleitete Schmerzen), sollte immer eine orientierende Untersuchung der angrenzenden anatomischen Bereiche erfolgen. Bei Kniegelenkschmerzen betrifft dies insbesondere das Hüftgelenk, dessen Beweglichkeit orientierend geprüft werden muss, um eine behandlungsbedürftige Koxarthrose nicht zu übersehen. Auch ist eine sorgfältige Erhebung des neurologischen Status (Sensibilität und Motorik) und der Durchblutungsverhältnisse (Fußpulse, A. poplitea) erforderlich.

\section{Röntgenuntersuchung}

Die Basisdiagnostik bei Kniegelenkbeschwerden besteht aus einer Röntgenaufnahme mindestens in zwei Ebenen: Die a.-p.-Aufnahme sollte im Stehen erfolgen, da sich unter Belastung die tatsächliche Gelenkspaltverschmälerung als Ausdruck des Knorpelverlustes besser abbildet. Die zweite Ebene wird zunächst immer als seitliche Aufnahme (im Liegen) angefertigt. Zur Darstellung des Femuropatellargelenkes kann eine axiale Aufnahme der Patella hilfreich sein, die eine gesonderte Beurteilung von medialer und lateraler Patellafacette ermöglicht.

Bei der Auswertung des erstellten Röntgenbildes achtet man auf die Röntgenzeichen der Arthrose als Ausdruck eines Knorpelschadens (Gelenkspaltverschmälerung, subchondrale Sklerosierung, Osteophyten und Geröllzysten). Die frühe Gonarthrose deutet sich oft durch eine vermehrte subchondrale Sklerosierung noch vor einsetzender Gelenkspaltverschmälerung und winzige Osteophyten an der Eminentia intercondylaris an. Bei fortgeschrittenen Gonarthrosen kann es auch zur Darstellung von Schliffflächen, Kapselosteomen und Dezentrierung kommen. Der röntgenologisch nachweisbare Schweregrad von Arthrosen kann mit unterschiedlichen Klassifikationssystemen zur Einteilung degenerativer Gelenkerkrankungen beschrieben werden. Am häufigsten kommt der Kellgren \& Lawrence-Score zur Anwendung, der von Grad 0 (keine Arthrosezeichen) bis Grad 4 (schwerste Arthrose) reicht (Tab. 2) [14].

\section{Schnittbildverfahren}

Schnittbildverfahren (CT, MRT) sind allenfalls ergänzende Untersuchungsmaßnahmen nach einer primär durchzuführenden konventionellen Röntgenaufnahme. Sie können in Einzelfällen zur weitergehenden Beurteilung von nicht knöchernen Gelenkstrukturen (z. B. Meniskusläsionen) oder zur differenzialdiagnostischen Abklärung (z.B. freie Gelenkkörper, Tumoren) indiziert sein. Auch sind mit geeigneten Sequenzen morphologische Knorpelveränderungen zunehmend besser diagnostizierbar. [7, 15].

\section{Differenzialdiagnostik}

Eine Vielzahl intra- und extraartikulärer Schmerzursachen muss differenzialdiagnostisch in Erwägung gezogen werden (Tab.3). Häufig ist eine Abgrenzung von Gelenkbeschwerden gegenüber ausstrahlenden Schmerzen aus anderen Regionen (z. B. Koxarthrose oder degenerative Wirbelsäulenerkrankungen) erforderlich. Hier kann die intraartikuläre Injektion eines Lokalanästhetikums zur Klärung beitragen.

Wichtig ist die Unterscheidung gegenüber entzündlich-rheumatisch bedingten Erkrankungen. Die Gonarthrose ist primär eine Erkrankung des Gelenkknorpels und entzündliche Phänomene (Gelenkerguss, Synovialitis) stellen nur eine Reaktion auf Abbauprodukte der zugrunde liegenden Knorpeldegradation dar. Bei den Erkrankungen des rheumatischen Formenkreises gilt dagegen die Entzündung der Synovialmembran mit Produktion kataboler Enzyme als die Hauptursache der sekundär folgenden Knorpelschädigung.

\section{Prognose und Ergebnisse}

Die individuelle Prognose zum Verlauf einer Gonarthrose ist sehr unsicher, da sich das Beschwerdebild phasenweise verändert und die Geschwindigkeit der weiteren Gelenkzerstörung durch eine Vielzahl meist noch unbekannter Faktoren beeinflusst wird. Zwar stehen in der konservativen Behandlung eine Vielzahl von beschwerdelindernden Maßnahmen zur Verfügung, doch gelingt es bisher nicht, die Progredienz wirksam aufzuhalten bzw. die Erkrankung zu heilen. Bei fehlender Möglichkeit zu achskorrigierenden Eingriffen und Versagen konservativer Behandlungsmaßnahmen ist relativ häufig nach mehrjähriger
Schmerzdauer letztlich die Implantation einer Endoprothese erforderlich. Im Verlauf der Erkrankung sollte man sich jedoch immer wieder kritisch die Frage stellen, ob Risikofaktoren identifizierbar sind, die möglicherweise beeinflusst werden können, um den Verlauf der Erkrankung günstig zu beeinflussen.

Tab.2 Röntgenologische Klassifikation des Arthrose-Schweregrads (Kellgren und Lawrence 1963) [13]

\begin{tabular}{ll}
$\begin{array}{l}\text { Schwere- } \\
\text { grad }\end{array}$ & $\begin{array}{l}\text { Röntgenologischer } \\
\text { Befund }\end{array}$ \\
\hline Grad 0 & keine Arthrosezeichen \\
\hline Grad 1 & $\begin{array}{l}\text { fraglicher Nachweis von } \\
\text { Osteophyten }\end{array}$ \\
\hline Grad 2 & $\begin{array}{l}\text { sicherer Nachweis von Osteo- } \\
\text { phyten, keine GSV }\end{array}$ \\
\hline Grad 3 & mäßige GSV \\
\hline Grad 4 & $\begin{array}{l}\text { Gelenkspalt erheblich ver- } \\
\text { schmälert bzw. aufgehoben }\end{array}$ \\
\hline
\end{tabular}

Tab.3 Wichtige Differenzialdiagnosen der Gonarthrose

Osteochondrosis dissecans, avakuläre Nekrosen

Weichteilerkrankung (z. B. Meniskusschaden, Bursitis)

Erkrankungen des rheumatischen Formenkreises

Septische Arthritis

Kristallarthropathien (z. B. Gicht)

Trauma

Tumorerkrankung

Nervenkompressionssyndrome

Erkrankungen von Wirbelsäule und Bandscheiben

Erkrankungen innerer Organe („referred pain")

Periphere arterielle Verschlusskrankheit 


\section{Fallbeispiele mit Bildern}

28-jährige Pat. mit Osteochondronekrose des lateralen Femurkondylus bei Diabetes mell. Typ 1 mit diabetischer Polyneuro-, Nephro- und Retinopathie Z.n. auswärtiger Refixation des osteochondralen Fragmentes und später ME mit Debridement (Abb.2).
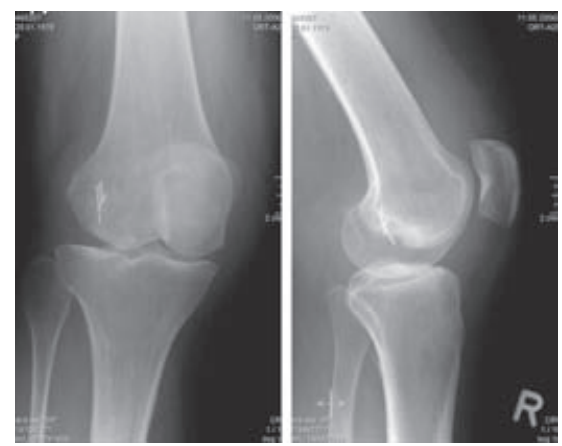

Abb. 2

71-jährige Pat., posttraumatische Valgusgonarthrose bei Z.n. lateraler Tibiakopffraktur vor $1 \mathrm{Jahr}$ (Abb.3-5).
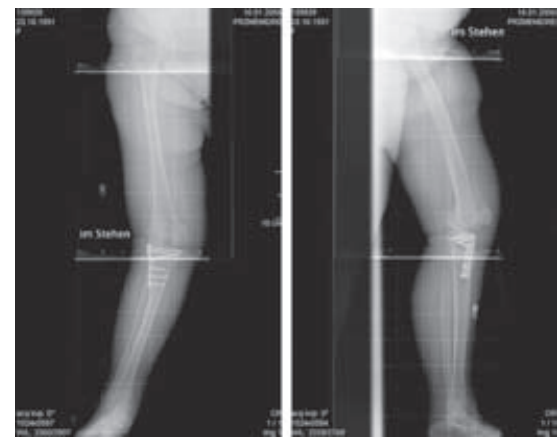

Abb.3 Ganzbeinaufnahme

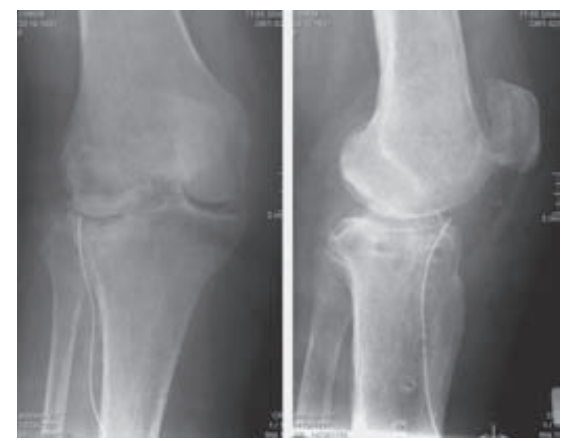

Abb. 4 nach ME 45-jährige Pat. mit idiopathischer Valgusfehlstellungbds.,rechtsZ.n.varisieren-
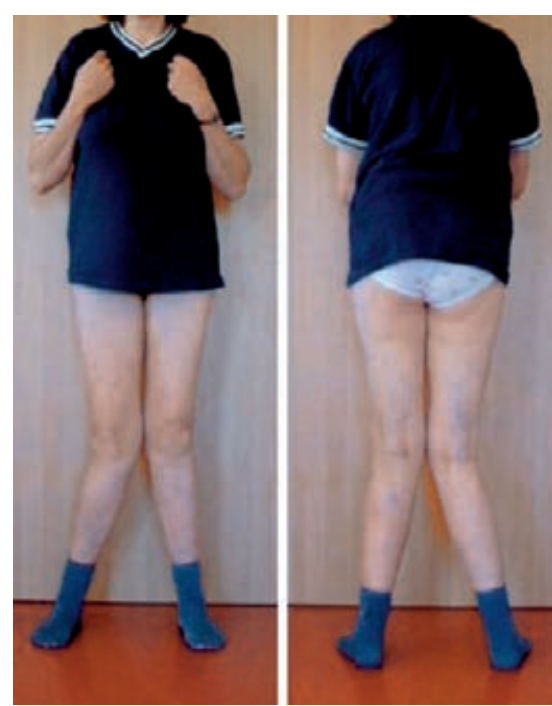

Abb.6 Klinisches Bild der Korrekturosteotomie vor 12 Jahren, Valgusgonarthrose bds. (Abb.6 und 7).

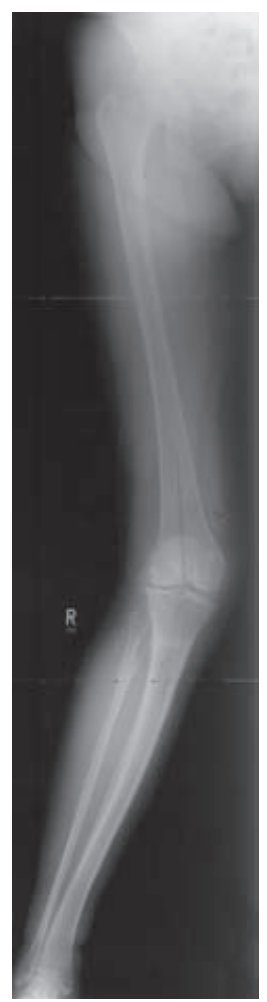

Abb. 7 Ganzbeinaufnahme rechts.

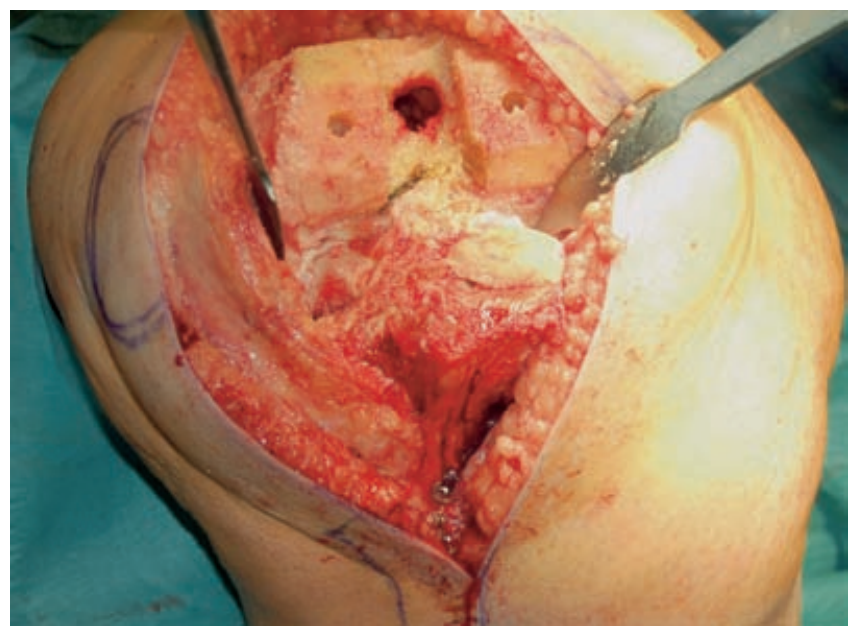

Abb. 5 intraoperativer Befund: Defekt laterales Tibiaplateau. 


\section{Literatur}

1 Aigner T, Rose J, Martin J, Buckwalter J. Aging theories of primary osteoarthritis: from epidemiology to molecular biology. Rejuvenation Res, 2004; 7(2): 134-145

2 Bellamy N. Buchanan W. W, Goldsmith C. H, Campbell J, Stitt. L. W. Validation study of WOMAC: a health status instrument for measuring clinically important patient relevant outcomes to antirheumatic drug therapy in patients with osteoarthritis of the hip or knee. J Rheumatol, 1988; 15(12): 183340

3 BQS: BQS Qualitätsreport 2005: Knie-Totalendoprothesen-Erstimplantation. In http:// www.bqs-qualitaetsreport.de/Webs/bqs/ qualrep/2005/ergebnisse/. Edited, 2006.

4 Brandt K. D, Lohmander S, Doherty M. The concept of osteoarthritis as failure of the diarthrodial joint. In: Bandt, K. et al (Hrsg.): Osteoarthritis. New York: Oxford University Press Inc 1998; 70-74

5 Brandt K. D, Mankin H. J, Shulman L. E. Workshop on etiopathogenesis of osteoarthritis. J Rheumatology 1986; 13: 1126-1160

6 Buckwalter J. A, Mankin, H. J, Grodzinsky, A. J. Articular cartilage and osteoarthritis. Instr Course Lect 2005; 54: 465-480

7 Gold G. E, Burstein D, Dardzinski B, Lang P, Boada F, Mosher T. MRI of articular cartilage in OA: novel pulse sequences and compositional/functional markers. Osteoarthritis Cartilage, 14 Suppl A: 2006; A76-86
8 Gross A. F, Fickert S, Gunther K. P. Übergewicht und Arthrose. Orthopäde 2005; 34(7): 638-644

${ }^{9}$ Günther K. P, Merx F. Arthrose. Bundesgesundheitsbericht, 2006 im Druck

10 Günther K. P, Stürmer T, Sauerland S, et al. Prevalence of generalised osteoarthritis in patients with advanced hip and knee osteoarthritis: the Ulm Osteoarthritis Study. Ann Rheum Dis 1998; 57(12): 717-723

11 Günther K. P, Stürmer T, Trepte C. T, Naumann T, Kinzl L, Puhl W. Häufigkeit gelenkspezifischer Risikofaktoren bei Patienten mit fortgeschrittenen Cox- und Gonarthrosen in der Ulmer Osteoarthrose-Studie. Z Orthop Ihre Grenzgeb 1999; 137(6): 468-473

12 Hackenbroch M. Die Arthrosis deformans. Grundlagen und Behandlung. Leipzig: Thieme, 1943

${ }^{13}$ Kellgren J. H, Lawrence J. S. Atlas of standard radiographs of arthritis. Oxford: Blackwell Scientific Publications, 1963.

${ }^{14}$ Kellgren J. H, Lawrence J. S. Radiological assessment of rheumatoid arthritis. Ann Rheum Dis 1957; 16(4): 485-493

${ }^{15}$ Lang P, Noorbakhsh F, Yoshioka H. MR imaging of articular cartilage: current state and recent developments. Radiol Clin North Am 2005; 43(4): 629-639

16 Stürmer T. Ulmer Osteoarthrose Studie. Z Orthop Ihre Grenzgeb 1998; 136(5): 11

17 Stürmer T, Brenner H, Brenner R. E, Gunther K. P. Non-insulin dependent diabetes mellitus (NIDDM) and patterns of osteoarthritis.
The Ulm osteoarthritis study. Scand J Rheumatol 2001; 30(3): 169-171

18 Stürmer T, Gunther K. P, Brenner H. Obesity, overweight and patterns of osteoarthritis: the Ulm Osteoarthritis Study. J Clin Epidemiol 2000; 53(3): 307-313

19 Sun Y, Stürmer T, Günther K. P, Brenner H. Inzidenz und Prävalenz der Cox- und Gonarthrose in der Allgemeinbevölkerung. Z Orthop Ihre Grenzgeb 1997; 135(3): 184-192

20 van Saase J. L, van Romunde L. K, Cats A, Vandenbroucke J. P, and Valkenburg H. A. Epidemiology of osteoarthritis: Zoetermeer survey. Comparison of radiological osteoarthritis in a Dutch population with that in 10 other populations. Ann Rheum Dis 1989; 48(4): 271-280

\section{Dr. med. Jörg Lützner}

Facharzt für Orthopädie

Dr. med. Stephan Kirschner Geschäftsführender Oberarzt Prof. Dr. med. Klaus-Peter Günther Klinikdirektor

Universitätsklinikum

Carl Gustav Carus Dresden

Orthopädische Klinik

Fetscherstraße 74

01307 Dresden 